\title{
Phase field modelling of irradiated materials
}

\author{
D. Simeone,${ }^{1}$ J. Ribis, ${ }^{1}$ and L. Luneville ${ }^{2}$ \\ ${ }^{1}$ DEN-Service de Recherches Metallurgiques Appliquees, \\ CEA, Universit Paris-Saclay, F-91191, Gif-sur-Yvette, \\ France CEA/CNRS/CentraleSupelec/LRC CARMEN 3 Rue Joliot Curie, 91190 Gif-sur-Yvette \\ ${ }^{2}$ DEN-Service d'Etude et de Recherches Mathematiques Appliquees, \\ CEA, Universit Paris-Saclay, F-91191, Gif-sur-Yvette, \\ France CEA/CNRS/CentraleSupelec/LRC CARMEN 3 Rue Joliot Curie, 91190 Gif-sur-Yvette
} (Dated: July 5, 2018)

\begin{abstract}
Almost forty years after Turings seminal paper on patterning, progress on modeling instabilities leading to pattern formation has been achieved. The initial concept of dissipative structure is now clearly understood within the Phase-Field framework. So far, such an approach obtained promising results in various aspects of materials research from pattern formation during solidification to defect dynamics. In this work, we will try discussing experimental results observed during aging of solids under irradiation within this framework. The approach followed in this presentation is comprehensive and not specialized in specific aspects of the Phase-Field modelling (mechanics, mathematics, or numerical methods) at the expense of a holistic picture.
\end{abstract}

\section{INTRODUCTION}

Materials exposed to neutron, electron or ion fluxes undergo a multitude of complex chemical and structural changes [1] at length scale spanning the atomistic to the macroscopic regimes over time scales raging from few pico-seconds to years. Predicting mechanical and thermal properties of these materials is difficult as irradiation drives the system away from equilibrium. Thus, the structural evolution of these materials under irradiation has been an ongoing field of research for several decades. Since it can induce variation of the local composition at the atomic scale, irradiation offers the unique opportunity to overcome the thermodynamic phase diagram manufacturing alloys with unexpected compositions fluctuation at the mesoscopic scale. On an academic point of view, materials under irradiation may be toy models to study the formation and the stability of systems maintained far from equilibrium. Varying the control parameter (temperature, particles flux), experimentalists can test different approaches for modeling systems far from equilibrium.

Over the past three decades, classical molecular dynamics (MD) methods were extensively applied to estimate primary damage production in materials (alloys and ceramics) submitted to radiation damage. This atomistic simulation methods allows to describe the first stage of radiation damage in materials. However, the time scale of the simulation (few pico-seconds) makes it unavailable to discuss long term aging of materials under irradiation over the diffusive time scale (few micro-second) that controls the micro-structure formation.

Different simulation techniques were developed over the past decades to predict the longtime micro-structural evolution of solids under irradiation. Usual approaches to model precipitates generated under irradiation (defects cavities, secondary phases and gas bubbles) during the aging of materials at the mesoscale, i.e. over few hundreds of nanometers, is based on mean field rate theory (RT). In the RT formalism, classical nucleation theory is applied to determine the nucleation rate while reaction rate theory is used to define the spatially averaged vacancy, interstitial and solute populations [2,3]. In these models, the dynamics of different populations is treated as spatially averaged equations, i.e. with averaged sink strength terms (the underlying micro-structural features are assumed frozen) and neglecting spatial correlations of point defects. To overcome this last limitation, Kinetic Monte-Carlo (KMC) methods were developed enabling the spatial resolution of individual class of defects (point defect, dislocations, cavities..). In radiation damage processes, elementary processes (thermal diffusion, ballistic exchanges) occur over time scales smaller (at least over three orders of magnitude) than the longtime evolution of point defects (Frenkel pairs), atomic species (ordering/disordering) and the micro-structure [4] (dislocation loops). Decoupling between these time scales insures that a new micro-structure can be treated as resulting from a Markov process. A microscopic master equation [5] can then be built to mimic the time evolution of the system under irradiation. Kinetic Monte Carlo simulation, integrating formally the master equation, appears to be a powerful tool to handle radiation induced microstructure. This simulation tool, very efficient at low temperature when the point defect concentration is low, can be extended to include many dynamics acting in parallel. The primary limitation of the KMC technique is the simulation cell size which dictates the observable cluster density. However, the main limitation of such an approach is that different dynamics must be added by hand and that atoms are constrained in a rigid lattice neglecting vibration enthalpy and entropy that plays an important role in the stability of materials. Within such an approximation, the evolution of a micro-structure based on 
pre-defined defects (jump frequencies and reaction activation energies are input parameters). An other severe limitation of this technique comes from the act that lattice relaxations, i.e. relaxations induced by electric fields (for insulators) and/or elastic fields (for metals and alloys) can not be handled due to the limitation of the simulation boxes sizes.

Continuum methods such as the Phase Field (PF) methods are based on a mean field theory describing the evolution of the micro-structure by continuous fields evolving in space and time and then satisfying defined partial differential equations. The main advantage of this approach results from the fact that all spatial information on the micro-structure is contained in these fields. Concentration[6] (or more largely Order Parameter [7]) fields resulting from a coarse grained average of the atomic occupation over a discrete underlying rigid lattice simulate only the long range order of the systems like in the RT. However, correlations between these means values are introduced "by hand" via differential operators in a free energy functional (the so called Ginzburg term [7]). The main interest of this approach is its ability to take into account in-homogeneous local long range fields like strain fields occurring during the micro-structure evolution. The main limitation of such an approach is due to the absence of clear coarse grained procedures [8].

Although continuous approximations suffer from some limitations, they offer the unique opportunity to discuss self organization in condensed matter at equilibrium and far from equilibrium [9-11]. This works discusses radiation induced patterning in alloys modeled within the PF approximation pointing out the its ability to predict the morphology of the microstructure as well as the solubility limits without evoking the concept of effective temperature [12].

\section{PHASE FIELD APPROACH}

The Phase Field method is a extension of the Landau Theory of Phase Transition [13]. In its seminal work, Landau pointed out that phase transitions are associated with symmetry breaking and he defines the notion of Order Parameter (OP, null in the high symmetric phase) [7]. For instance in para-magnetic to ferromagnetic transition, the rotational symmetry of the magnetization vector (the OP) is broken in the ferromagnetic phase leading to domains of well defined orientation. To go a step beyond this approach, he intuited that the free energy of the system (more generally the thermodynamic potentials) are only function of the OP related with Long Range order in materials. The main drawback of the Landau's theory is to neglect the fluctuation (the evolution of the Short Range Order). In this sense, the Phase Field (PF) approach is a mean field approximation. To introduce spatial variation of the OP, named $\eta(\mathbf{x})$ below, local extra terms of the type $\nabla^{p}(\eta(\mathbf{x}))$ were introduced in the Free energy expression (Ginzburg term). The PF is a generalization of the Landau Theory dealing with the kinetic evolution of the OP $\eta(\mathbf{x}, t)$. Contrary to the rate theory extensively used to discuss formation of extended defects (voids, dislocations...) under irradiation, all information on the micro-structure is dictated by the variation of the Free energy (more precisely its first variation $\left.\frac{\delta F}{\delta \eta(\mathbf{x}, t)}\right)$ term without any need to external inputs like the emission or the absorption terms associated with the migration of point defects in the Rate Theory.

\section{Gradient and non gradient dynamics}

Assuming that the evolution of a system can be modelled within the PF framework, the variation of the Long Range Order (LRO) $\frac{d \eta(\mathbf{x})}{d t}$ is assumed to be linked to the variation of the Free energy functional $\frac{\delta F(\eta(\mathbf{x})}{\delta \eta(\mathbf{x})}$ :

$$
\begin{aligned}
\frac{d \eta(\mathbf{x}, t)}{d t} & =F(\eta(\mathbf{x}, t), \mu) \\
& =F_{R}(\eta(\mathbf{x}, t), \mu)+F_{N R}(\eta(\mathbf{x}, t), \mu)
\end{aligned}
$$

$F$ was separated in two distinct terms. The first one $F_{R}(\eta(\mathbf{x}, t), \mu)$ is associated with the relaxation part $-\Gamma \frac{\delta \mathcal{L}}{\delta \eta(\mathbf{x}, t)}$ of the dynamics $(\mathcal{L}$ is a real function and $\Gamma$ is a positive operator). $F_{N R}(\eta(\mathbf{x}, t), \mu)$ describes all non relaxing processes associated with the dynamics and is orthogonal to $F_{R}(\eta(\mathbf{x}, t), \mu)$ for a given inner product [9].

Different cases exist:

- If $F_{N R}=0$ and $\Gamma$ is constant. The evolution of the system follows the line of steepest descent of $\mathcal{L}$. The system is named as a relaxation gradient flow

- If $F_{N R}=0$ and $\Gamma$ are not constant (system with conservation laws), transient patterns do not correspond to the lines of steepest descent of $\mathcal{L}$ can appear. The system is a relaxation non-gradient flow.

- if $N$ is non null, the status of the dynamics is still under investigation. The system is a non relaxation potential flow.

- $F$ reduces to a constant and nothing can be said about the long term evolution of the system. This system is a non potential flow.

For systems described by relaxation gradient dynamics out of equilibrium,i.e. modelled as a diffusion process, their behavior should be similar to those at equilibrium since the Lyapounov functional plays the same role than the free energy and can be understood as an "effective free energy". This analysis is the key point of the continuum modelling of materials under irradiation. In this approach, the complex external dynamics induced by slowing down of impinging particles in the solid reduces to 
a diffusion-like process defined by a a-thermal diffusion coefficient. The total dynamics is then described as a relaxation gradient flow and this dynamics is governed by the evolution of a Lyapounov functional $\mathcal{L}$ which plays the role of an effective "thermodynamic potential" far from equlibrium. If $\mathcal{L}$ can be computed, the long term behavior of the microstructure caz be determined.

In particular, spatio-temporal patterns should appear and generate Turing-like patterns leading to the formation of topological defects beyond Turing-like instabilities. The next section is devoted to study the non equilibrium behavior of radiation induced decomposition in non miscible alloys within the PF framework.

\section{Spinodal decomposition of alloys under irradiation}

The spontaneous formation of patterns under irradiation was observed in many non-miscible alloys for seventy years. The observed experimental patterns result from the existence of steady states. The main ingredient of this approach is the competition between a thermally activated dynamics driving the system at temperature $T$ to the equilibrium and an externally imposed particles exchange $\Gamma^{e x t}(\phi)$ regardless of the chemical identity of particles, by essence a-thermal $(T=\infty)$ and driven by the nature, the energy and the flux $\phi$ of impinging particles. Martin [12] points out that the ordering induced under irradiation is indistinguishable from that of an equilibrium Ising model assuming that this exchange has an infinite range as expected [14]. The connection between these dynamical systems and equilibrium system is performed via the construction of a Lyapounov functional acting as an effective free energy. Thus, the dynamics of orderdisorder transformation under irradiation for binary alloys with positive heat of mixing is indistinguishable to an equilibrium Cahn-Hilliard $(\mathrm{CH})$ equation, the continuum counterpart of the Ising model, with infinite-range interactions. By analogy with the $\mathrm{CH}$ equation including elastic energy assumed to be of infinite-range [6, 15], a "law of corresponding states" can be introduced for a system at temperature $T$ via $T_{\text {eff }}=T(1+\Delta)$. $T_{\text {eff }}$ can be understood as the effective temperature of the system and $\Delta=\frac{\Gamma^{e x t}(\phi)}{\Gamma(T, \phi)}(\Gamma(T, \phi)$ is the thermally activated frequency of atoms promoting equilibrium) is a kinetic enhancement factor induced by the infinite range exchanges. From this analysis, $T_{\text {eff }}$ becomes important at very low irradiation temperatures and do not affect the stability of precipitates at high temperature and low doses in agreement with experimental results [16]. Since a-thermal exchanges are assumed to take place over an infinite range, such an approach is also unable to describe patterns associated with spatial modulation of the chemical composition of the alloy at the mesoscopic scale, i.e. few tens of nanometers, experimentally observed un- der irradiation [17]. To overcome this difficulty, the exchange of atoms occurring during the irradiation process is assumed to occur over a finite-range with an average relocation distance $R$ by analogy with phase separation induced by long range Coulomb interactions describing similar patterns [18].

At equilibrium, the local composition (atomic fraction) given by the order parameter $\eta(\mathbf{x}, t)=c(\mathbf{x}, t)-c_{0}$, null in the disordered high temperature phase of uniform concentration $c_{0}$ in the $A_{\bar{c}} B_{1-\bar{c}}$ alloy of average composition $\bar{c}$ is dictated by the $\mathrm{CH}$ equation routinely employed to study the phase separation dynamics within the PF framework:

$$
\frac{\partial \eta(\mathbf{x}, t)}{\partial t}=M \nabla^{2}\left[\frac{\delta\left[\int f(\eta(\mathbf{x}, t)) d \mathbf{x}\right]}{\delta \eta(\mathbf{x}, t)}\right]
$$

where $f(\eta(\mathbf{x}, t))$ the free energy density results from a fourth power Landau polynomial expansion $f(\eta(\mathbf{x}, t))=$ $\frac{a_{2}}{2} \eta(\mathbf{x}, t)^{2}+\frac{a_{3}}{3} \eta(\mathbf{x}, t)^{3}+\frac{a_{4}}{4} \eta(\mathbf{x}, t)^{4}$, the parameters of which are fitted to satisfactorily reproduce the equilibrium phases [19].

To introduce the spatial variation of $\eta$, a Ginzburg term $\int \frac{\left|a_{2}\right| \xi}{2}|\nabla \eta(\mathbf{x}, t)|^{2} d \mathbf{x}$ is added to the free energy. The term $\xi$ is related to interfaces thickness [7]. In this equation, $\mathrm{M}$ is the averaged mobility of species assumed to be concentration independent [20]. The contribution of irradiation to the kinetic evolution of the alloy composition entering Eq. (2) can be expressed by the following equation $[21,22]$ :

$$
\begin{aligned}
\left(\frac{\partial \eta(\mathbf{x}, t)}{\partial t}\right)^{e x t} & =\Gamma^{e x t}(\phi)\left[\int p_{R}(\mathbf{x}-\mathbf{y} \eta(\mathbf{y}, t) d \mathbf{y}-\eta]\right. \\
& =\Gamma^{e x t}(\phi)\left[<\eta>_{R}-\eta\right]
\end{aligned}
$$

where $p_{R}(\mathbf{x} \propto \exp (-|x| / R))$ is the density probability function describing the relocation of atoms set in motion by elastic collisions. By analogy with the studies of long range interactions at equilibrium [18, 23], the radiation induced dynamics can be written as:

$$
\begin{aligned}
\frac{\partial \eta(\mathbf{x}, t)}{\partial t}{ }^{e x t} & =\frac{\Gamma^{e x t}(\phi)}{2} \frac{\delta\left[\int d \mathbf{x} d \mathbf{y} \eta(\mathbf{x}, t) G(\mathbf{x}, \mathbf{y}) \eta(\mathbf{y}, t)\right]}{\delta \eta} \\
& =\frac{\Gamma^{e x t}(\phi)}{2} \frac{\delta\left[\int d k \hat{G}(k)|\eta(\mathbf{k}, t)|^{2}\right]}{\delta \eta(-\mathbf{k}, t)}
\end{aligned}
$$

where $G(\mathbf{x}, \mathbf{y})=G(|\mathbf{x}-\mathbf{y}|)$ expresses the spatial dependence of the radiation induced relocation and can be understood as an effective long range interaction. The total dynamics resulting from the evolution in parallel of the thermal and the irradiation dynamics is a relaxation gradient flow associated with a Lyapounov $\mathcal{L}[\eta]=F\left(\eta\left((x, t)+\frac{\Delta}{2}\left(\int \eta(\mathbf{x}, t) G(\mathbf{x}, \mathbf{y}) \eta(\mathbf{y}, t) d \mathbf{x} d \mathbf{y}\right)\right.\right.$.

Fig 1 displays the 2D long term evolution of the OP $\eta((x), t)$ resulting from a PF simulation. The Power spectrum of the micro-structure, i.e. the structure factor of 


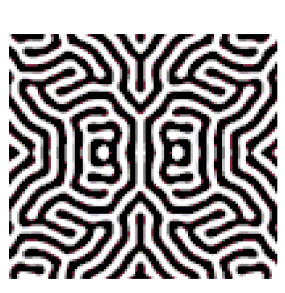

(a) $\mathrm{t}=1000$

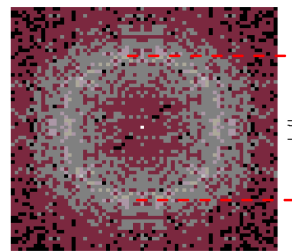

(b)

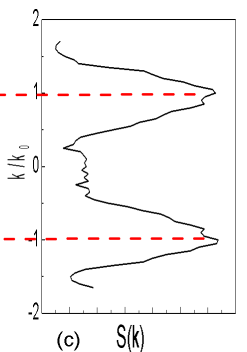

(c) $S(k)$
FIG. 1. (a) 2D numerical simulation of the long time microstructure (1000 in reduced units) of $\mathrm{AgCu}$ alloy irradiated by $1 \mathrm{Mev} \mathrm{Kr}$ ions. Black (white) domains corresponds to $\mathrm{Cu}$ rich (poor) regions $(c \geq 0.5$ and $c \leq 0.5$ respectively. Boundaries conditions implies that the calculated micro-structure exhibit a point symmetry and two mirror symmetries. (b) Power spectrum of the micro-structure. This Power spectrum, i.e. the structure factor of the OP exhibits a Mexican hat shape. The maximum of its intensity exists for a well defined modulus $k_{0}=\sqrt{k_{x}^{2}+k_{y}^{2}}$ of waves vectors as displayed in figure c.

$\eta((x), t)$, exhibits a ring shape for long times revealing the existence of wave vector modulations of modulus $k_{0}$.

A careful inspection of $\mathcal{L}$ clearly shown that order disorder transitions $\left(k_{0}=0\right)$ or a decomposition process occurs (non null $k_{0}$ values) for different $(\Delta, R)$ couples as displays in Fig 2. This analysis is in perfect agreement with results of numerical calculation [24] showing the evolution of the structure factor in the pattering region (Figure 2). From the analysis of different factors structures, it becomes possible to compute the evolution of $k_{0}$ versus $R$ for given $\Delta$ values as displayed in Figure 2. This evolution extracted from full PF simulation shows a clear linear evolution of $k_{0}$ with $R^{-1}$ in agreement with theoretical investigations [25].

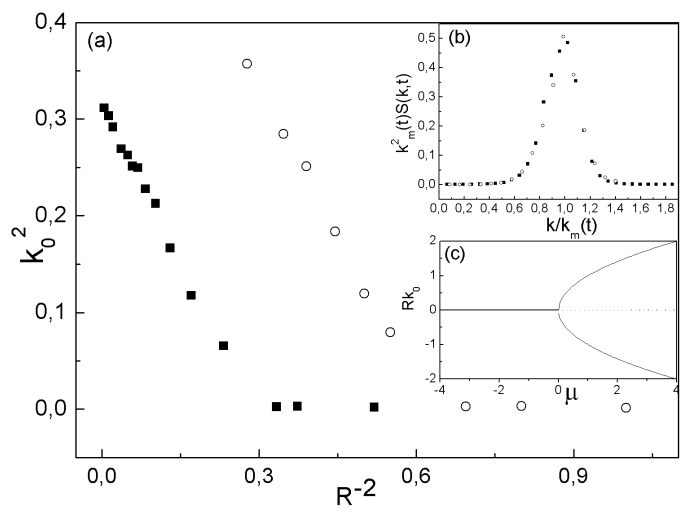

FIG. 2. (a) Evolution of $k_{0}$ versus $R$ for different $\Delta$ values (black squares: $\Delta=0.04$, open circles: $\Delta=0.7$ ), (b) Values of $k_{0}$ are extracted from universal structures factor extracted from PF simulations. (c) the evolution of $k_{0}$ versus the control parameter $\mu=\sqrt{\Delta R^{4}}-1$ displays a super-critical bifurcation.

Patterns produced under irradiation result from slow composition modulations in space and time of simple basic patterns defined by the wave vector of modulus $q_{0}$ minimizing $\mathcal{L}$. Although each individual stationary pattern breaks transitional and rotational symmetries (Fig 3), different patterns grow in different parts of the system implying the formation of various defects connecting these different steady states [9] as displayed in Fig 3. From the stationarity of $\mathcal{L}$, it is possible to compute all possible micro-structures produced in a pattern domain as a function of the overall concentration of the alloys $(\bar{\psi}$ in reduced unit) as well as a function of $\Delta$ and $R$ noted $\epsilon$ as displayed in Fig.3.

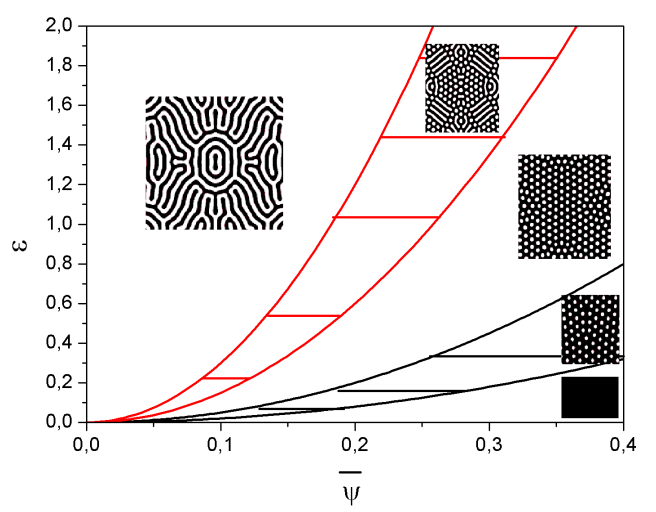

FIG. 3. 2D phase diagram for steady states obtained from $\mathcal{L}_{\mathcal{S H}}(t)$ in the one mode approximation. Depending on the averaged composition ( $\bar{\psi}$ in reduced units), stripes, bubbles in hexagonal networks and constant phase can be created. Hatched areas correspond to co-existence domains. This phase diagram is validated by numerical simulations (micro structures drawings). All simulations were performed for $\Delta=0.2, R=3, t=3000$ and the domain size equal to 200 in reduced units.

From the knowledge of this "pseudo phase diagram", it becomes possible to determine the solubility limits of precipitates produced under irradiation (black dots in Fig 3). The minimization of the Lyapunov functional for given micro-structures leads to an analytical formulation ofthe solubility limits of radiation induced precipitates. These formulae clearly highlight that solubility limits also depend not only on irradiation parameters $\phi$ and $T$ but also on the average composition. Fig 4 displays the comparison between the solubility limits calculated from the preceding equations and resulting from direct computations of the totoal dynamics ( Eq. 2 and Eq. 3). Three distinct domains are clearly visible in Fig 4 . In the first domain (I) where the spinodal decomposition occurs, the solubility limits result from the minimization of the full Lyapounov functional and evolve like $\sqrt{1-\Delta R^{2}}$ (black lines in Fig 4). In the third domain (III) where the patterning occurs, solubility limits extracted from direct simulations are similar to analytically ones resulting from the minimization of the Lyapounov within the one mode approx- 
imation. A discrepancy between solubility limits calculated from the minimization of the Lyapunov functional and extracted from numerical simulations clearly appear in the second domain (II). This discrepancy results from the fact that the one mode approximation does not hold in this domain. It can be pointed out from these results that none of these solubility limits can be calculated applying the effective temperature concept.

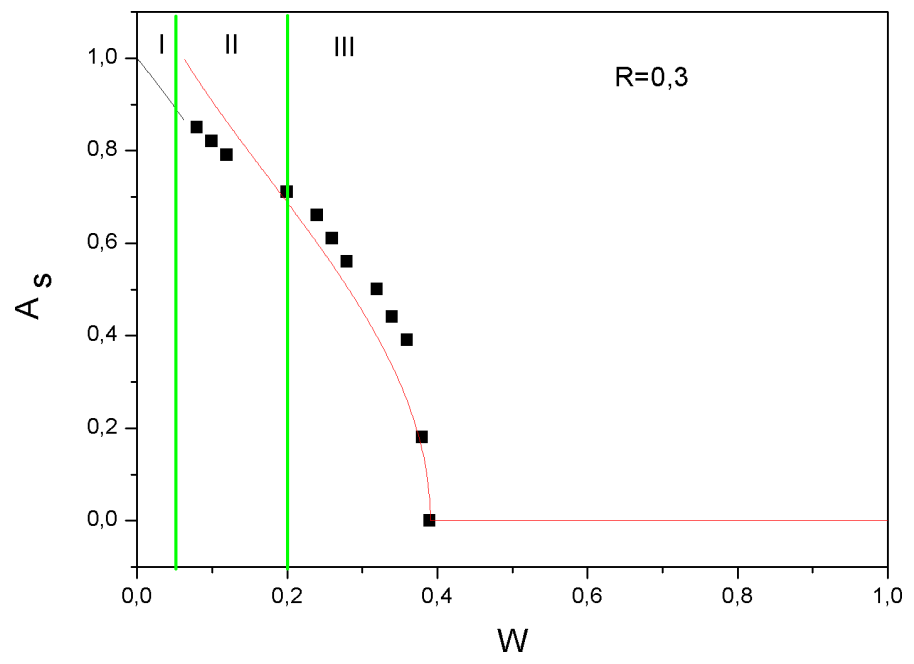

FIG. 4. Solubility limits $A_{s}$ (lines) extracted from minimization of The lyapounov functional and from direct numerical simulations (black squares) in the patterning domain. This graph highlights three distinct domains. In the first domain (I), the solubility limits results from the minimization of a labyrinthine micro-structure (stripes) as expected for a spinodal decomposition (black line). In the third domain (III), the solubility limits derived from the one mode approximation (full red line) is in fair agreement with the numerical results (red line). In the second domain (II), the one mode approximation fails to reproduced numerical results because the one mode approximation used to minimize the Lyapunov functional does not hold.

\section{Radiation induced amorphization}

An amorphous phase can be assumed to result from a phase transition from a parent crystal phase. Such an assumption can explain the observed experimental irradiation or pressure induced amorphizations. For glasses resulting from a liquid phase, the crystal parent phase is bypassed on over-cooling since the local glass structure generally reflects the chemical bonding of the bypassed crystal [26]. Following the theory of incommensurate structures, the critical wave vector describing the crystalamorphous transition varies continuously from one point to another in the amorphous state $\mathbf{k}_{\mathbf{c}}(\mathbf{x})=\sum_{k=1}^{3} \rho_{k}(\mathbf{x}) \mathbf{t}_{\mathbf{k}}^{*}$ where $\mathbf{t}_{\mathbf{k}}^{*}$ are reciprocal lattice vectors of the parent crystal phase $[27,28]$

Breaking the translation symmetry in the amorphous phase leads to the existence of an internal stress field associated with topological defects and function of the order parameter within the Phase Field framework. Such an approach may be useful to explain radiation induced amorphization observed in many alloys and ceramics [25, 29].

\section{CONCLUSION}

More than fifty years were devoted to show that irradiation of materials with energetic particles induces drastic changes in their micro-structure and may then induce important variation of their physico-chemical properties. This radiation response of materials is important in various technological fields ranging from nuclear industry to micro-electronics and surface engineering. The existence of an extensive experimental database in this area allows us to present a large perspective on the physical nature of self organization in irradiated materials. The large amount of defects produced under irradiation drives the system far from equilibrium.

The challenge in the prediction of radiation induced microstructure results from the integration of different mechanisms acting on different space and time scales in a unified framework. The long term evolution of these systems maintained far from equilibrium by slowing down of impinging particles can be understood as an example of reaction diffusion kinetics extensively used to described patterns formation in chemistry and biology. Adopting a continuum approach, we point in this work that the Phase field approach can capture main flavors of the long term evolution of the radiation induced micr-structure. The main interest of this approach is that it is self consistent, in the sense that all properties of the system under consideration are defined by a "free energy" like term only function of the Order Parameter at least for relaxation processes. On the other hand, this method offers the unique opportunity to handle long range interactions like elastic fields that play an important role in the patterns formation. Limitation associated with the use of phenomenological coefficients for this free energy like term can easily be overcome performing a multi-scale approach [19]. However, the main limitation of this approach is due to the lack of fluctuations associated with the kinetics, forbidding any discussion on the relative stability of steady states.

[1] V. Kuzovkov, E. Kotomin, G. Zvejnieks, K. Li, T. Ding, and L. Wang, Advances in Materials science research, ed. M Wythers, Nova Science Publishers 2, Chapter 11 (2011).

[2] E. Kotomin, V. Kuzovkov, and W. Von Niessen, Phys. Rev. B. 58, 8454 (1998). 
[3] E. Kotomin, M. Zaiser, and W. Soppe, Phil. Mag. A 70, 313 (1994).

[4] G. Zvejnicks, P. Merzlyakov, V. Kuzovkov, and E. Kotomin, Nucl. Instr. and Methods B 368, 138 (20168).

[5] T. Opplestrup, V. Bulatov, G. Gilmer, M. Kalos, and B. Sadigh, Rep. Prog. Phys. 55, 2079 (1992).

[6] A. G. Khatchaturyan, Theory of structural transformation in solids (Wiley Interscience, 1983).

[7] P. Tolédano and V. Dmitriev, Reconstructive phase transitions: in crystals and quasicrystals (World Scientific, 1996).

[8] K. Elder, M. Katakowski, M. Haataja, and M. Grant, Phys. Rev. Lett. 88, 245701 (2002).

[9] N. Ghoniem and D. Walgraef, Instabilities and self organization in materials, vol I (Oxford Science publications, 2008).

[10] G. Nicolis and I. Prigogine, Self organization in Nonequilibrium Systems: from dissipative structure to order through fluctuations (Wiley-NY, 1977).

[11] G. Van Kampen, Processes in Physics and Chemistry (Amsterdam-NH, 1980).

[12] G. Martin, Phys. Rev. B 30, 53 (1984).

[13] M. Le Bellac, F. Mortessagne, and G. Batrouni, Equilibrium and non equilibrium statistical thermodynamics (Cambridge University Press, 2010).

[14] B. Bergersen and Z. Racz, Phys. Rev. Lett. 67, 3047 (1991).

[15] E. L. Huston, J. W. Cahn, and J. E. Hilliard, Acta
Metall. 14, 1053 (1966).

[16] Y. Adda, M. Beyeler, and G. Brebec, Thin Solid Films 25, S28 (1975).

[17] B. Tsaur, S. Lau, and J. Mayer, Appl. Phys. Lett. 36, 823 (1980).

[18] L. Chen and A. Khatchaturyan, Phys. Rev. Lett. 70, 1477 (1993).

[19] G. Demange, L. Luneville, V. Pontikis, and D. Simeone, J. Appl. Phys. 121, 125108 (2017).

[20] A. J. Bray, J. Adv. Phys. 43, 357 (1994).

[21] P. Sigmund and A. Gras-Marti, Nucl. Instrum. Methods Phys. Res., Sect. B 182, 211 (1981).

[22] D. Simeone and L. Luneville, Phys. Rev. E 81, 021115 (2010).

[23] T. Ohta and K. Kawasaki, Macromolecules 19, 2621 (1986).

[24] L. Luneville, K. Mallick, V. Pontikis, and D. Simeone, Phys. Rev. E 94, 052126 (2016).

[25] D. Simeone, J. Costantini, L. Luneville, L. Desgranges, P. Trocellier, and P. Garcia, J. Mater. Res. 30(9), 2015 (2015).

[26] S. Karlin and H. M. Taylor, The Physics of Amorphous Solids (Wiley, NY, 1983).

[27] P. Toledano, Euro-physics Letter 78, 46003 (2007).

[28] A. Braginsky, Journal of experimental and theoretical physics 105, 30 (2007).

[29] C. Massobrio, V. Pontikis, and G. Martin, Euro-physics Letter 62, 1142 (1989). 